\title{
Histórias de crianças e adolescentes que (con)vivem com doença crônica
}

Stories of children and adolescents that (co)live with chronic disease

Historias de niños y adolescentes que (con)viven con enfermedad crónica

Jéssica Íris Franco da Silva1 (D) https://orcid.org/0000-0001-8220-2172

Jonas Sâmi Albuquerque de Oliveira $^{1}$ (D) https://orcid.org/0000-0003-0303-409x

Maria Lúcia Azevedo Ferreira de Macêdo ${ }^{1}$ (D) https://orcid.org/0000-0003-4923-4441

Ana Karina da Costa Dantas ${ }^{1}$ (D) https://orcid.org/0000-0002-7705-0554

Sandra Michelle Bessa de Andrade Fernandes ${ }^{1}$ (D) https://orcid.org/0000-0001-8441-1402

Francisca Andreza de Melo ${ }^{1}$ (D) https://orcid.org/0000-0002-6622-4723

\section{Resumo}

Objetivo: Revelar a história de vida de crianças e adolescentes que convivem com doença crônica, a partir de seus próprios relatos.

Métodos: Trata-se de um estudo descritivo de abordagem qualitativa. Utilizou-se a história oral temática como método e contou-se com o auxílio do software Atlas.ti 8.0 para organizar os dados.

Resultados: Oito participantes foram entrevistados. Quatro categorias foram identificadas: Modificações na vida; Ruptura escolar; Impactos e Perspectivas e sonhos. Revelou-se que a maior parte dos participantes conhece 0 diagnóstico da doença e reconhece as implicações dela em seu cotidiano, como as diversas restrições impostas por ela. Os participantes descreveram as modificações na rotina, citando necessidades específicas e de internação hospitalar, mas, apesar de tudo, também expressaram suas perspectivas para o futuro.

Conclusão: Foram identificados os sentimentos relacionados a doença crônica, sendo possível ter uma descrição de como é conviver com uma doença e, ao mesmo tempo, realizar as atividades da vida quotidiana.

\section{Abstract}

Objective: Reveal the life history of children and adolescents living with chronic illness, based on their own reports. Methods: This is a qualitative descriptive study. Thematic oral history was used as a method, and relied on the Atlas. ti 8.0 software to organize data.

Results: Eight participants were interviewed. Four categories were identified: Changes in life; School break; Impacts and Perspectives and dreams. It was revealed that participants know about the diagnosis and recognize its implications in their daily lives, as the various restrictions caused. They described changes in routine, citing specific needs and hospitalization, but nonetheless also expressed their prospects for the future.

Conclusion: Feelings related to chronic illness were identified. It was possible to understand their description of what it is like to live with the disease in performing activities of daily living.

\section{Resumen}

Objetivo: Revelar la historia de vida de niños y adolescentes que viven con enfermedades crónicas, en base a sus propios informes.

Métodos: Se trata de un estudio descriptivo. La historia oral temática se utilizó como método y se basó em el software Atlas.ti 8.0 para ayudar la organización de los datos.

Resultados: Se entrevistó a ocho participantes. Se identificaron cuatro categorías: Cambios en la vida; Vacaciones escolares; Impactos y Perspectivas y sueños. Se reveló que la mayoría de los participantes conocen el diagnóstico y reconocen sus implicaciones em su vida diaria, como lo causaron las diversas restricciones. Describieron cambios em la rutina, citando necesidades específicas y hospitalización, pero sin embargo también expresaron sus perspectivas para el futuro.

Conclusión: Se identificaron sentimientos relacionados con enfermedades crónicas, donde era posible tener una descripción de cómo es vivir con la enfermedad al realizar actividades de la vida diaria.

\section{Como citar:}

Silva Jl, Oliveira JS, Macêdo ML, Dantas AK, Fernandes SM, Melo FA. Histórias de crianças e adolescentes que (con)vivem com doença crônica. Rev Soc Bras Enferm Ped. 2021;21(2):65-71.

${ }^{1}$ Universidade Federal do Rio Grande do Norte, Natal, RN, Brasil.

Conflitos de interesse: nada a declarar.

Submetido: 2 de Fevereiro de 2020 | Aceito: 3 de Dezembro de 2021

Autor correspondente: Jéssica Íris Franco da Silva | E-mail: irisjessicafs@gmail.com

DOI: http://dx.doi.org/10.31508/1676-379320210010 


\section{Introdução}

As doenças crônicas estão inseridas no conjunto de condições crônicas e, em geral, relacionam-se com múltiplas causas, sendo caracterizadas por se iniciarem gradualmente, terem prognóstico incerto, além de terem duração prolongada ou indefinida. O curso clínico geralmente muda com o passar do tempo, podendo evoluir com períodos de agudização, assim como causar incapacidades em alguns casos. Essas condições ocasionam elevados números de internações hospitalares que envolvem intervenções de variadas naturezas, alterando o modo de vida dos indivíduos acometidos, num processo contínuo de cuidado que nem sempre leva à cura. ${ }^{(1)}$

Para cada fase da doença crônica em crianças e adolescentes, existem necessidades singulares, o que exige da Rede de Atenção à Saúde, assim como dos profissionais integrantes, certa sensibilidade para realizar o acolhimento por meio de um cuidado que seja apoiado, ampliado, contínuo, proativo e resolutivo, pois esses são aspectos fundamentais da atenção para a referida população. ${ }^{(2)}$

As doenças crônicas na infância impõem limitações à criança, sujeitando-a a procedimentos invasivos e longos períodos de internação. Em relação ao Brasil, estudos apontam que $49,1 \%$ das internações infantis são de crianças com doenças crônicas, enquanto as reinternações delas chegam a $50 \%$. $^{(3,4)}$

Com isso, tendo em vista a vulnerabilidade desse público e a necessidade da efetivação de uma gestão profissional para um cuidado mais ampliado para o manejo adequado da situação, também é preciso que a equipe multiprofissional possua um olhar mais ampliado como ferramenta de apoio em sua assistência, de forma a identificar as necessidades da criança ou adolescente, para além da condição biológica da doença, e de suas redes familiares e sociais. ${ }^{(5,6)}$

Conviver com uma doença crônica inevitavelmente inclui uma multiplicidade de contextos e de desafios, tanto para o indivíduo como para sua família, e essas diferentes variáveis podem facilitar ou dificultar essa vivência com o adoecimento. Estudos apontam que o bem-estar de crianças com enfermidades crônicas é condicionado à qualidade das experiências nesses contextos, como o relacionado ao diagnóstico e ao processo de hospitalização, por exemplo, em que os discursos e práticas dos profissionais podem ter profundo impacto na criança/adolescente. ${ }^{(6)}$

Além disso, reconhecendo que é importante explorar como as condições crônicas afetam o dia a dia desses indivíduos, seus relatos e narrativas são muito importantes para compreender profundamente como eles convivem com essa realidade e como se dá o manejo da doença. Segundo estudos, as crianças são as melhores fontes de informação sobre suas próprias experiências e opiniões, porém a maneira como se dão a comunicação e a expressão dos sentimentos está extremamente atrelada aos contextos físico, social, econômico e político delas. Nesse sentido, a partir de uma abordagem qualitativa, é possível averiguar os eventos ocorridos sob a visão das crianças, assim como o significado que elas conferem a essas vivências. ${ }^{(7)}$

A partir das experiências cotidianas no ambiente hospitalar, percebeu-se a fragilidade das crianças e adolescentes com doenças crônicas, assim como a de suas famílias, no tocante a conviver com os mais variados tipos dessas condições, além de todos os processos que as permeiam, como a descoberta, o manejo e o enfrentamento delas.

Diante do exposto, o presente estudo teve como objetivo revelar a história de vida de crianças e adolescentes que convivem com doença crônica, a partir de seus próprios relatos.

\section{Métodos}

Trata-se de um estudo descritivo, com abordagem qualitativa, que buscou revelar a história de vida de crianças e adolescentes que convivem com doença crônica.

Os dados foram coletados exclusivamente por uma enfermeira-residente em Saúde da Criança na Unidade de Terapia Intensiva Pediátrica e na Enfermaria Pediátrica do Hospital Universitário Onofre Lopes (HUOL), tanto à beira do leito, quando os participantes se encontravam impossibilitados de sair do leito, quanto na área de convivência da enfermaria, espaço destinado ao lazer dos pacientes hospitalizados e suas famílias. Esses locais foram escolhidos devido à metodologia do estudo, que requer um espaço cômodo e privativo, e a pesquisadora foi escolhida por ter vínculo prévio com os participantes da amostra, pois sabe-se a importância de estabelecer uma relação de 
confiança anterior, em especial quando se trata de público nessa faixa etária.

Participaram do estudo crianças e adolescentes com diagnóstico de doença crônica que estiveram em processo de internação hospitalar entre o período de maio e agosto de 2019.

Os critérios de inclusão foram: ser de ambos os sexos; ser criança ou adolescente; possuir qualquer doença crônica com diagnóstico médico sem tempo determinado; estar hospitalizado(a) na referida unidade; e ter capacidade física e cognitiva para corresponder aos objetivos da pesquisa.

Já referente aos critérios de exclusão, eles foram: pacientes que apresentassem comprometimento físico, neurológico e/ou cognitivo que os impossibilitassem de corresponder aos questionamentos da pesquisa e aqueles impossibilitados de corresponder às questões por qualquer circunstância não inclusa no critério anterior, como, por exemplo, aqueles com deficiência visual ou auditiva, traqueostomizados, entre outros.

Dado o conhecimento da rotina dos setores pela pesquisadora, o recrutamento dos participantes foi realizado de acordo com os horários de menor movimentação de terceiros. As crianças e adolescentes com seus acompanhantes foram abordados e informados de maneira clara, com linguagem acessível, sobre os aspectos da pesquisa. Foi exposto que a participação seria voluntária, e não obrigatória, que os dados fornecidos seriam confidenciais e anônimos, que eles poderiam desistir a qualquer momento, sem sofrer nenhum prejuízo na continuidade da assistência no serviço, e que a pesquisadora estaria disponível para esclarecer qualquer dúvida.

Para cada participante da pesquisa, foram solicitadas a leitura do Termo de Assentimento Livre e Esclarecido - ou foi lido para ele(a) - e, em caso de concordância com o conteúdo, a assinatura dele; também foi solicitada a assinatura do responsável legal no Termo de Consentimento Livre e Esclarecido, sob as mesmas condições. Não houve nenhuma recusa no decorrer da pesquisa.

Utilizou-se a história oral temática descrita por Meihy como método para obtenção dos dados. ${ }^{(8)}$ As intervenções foram realizadas individualmente, preservando-se a espontaneidade dos participantes. Foi utilizado um roteiro de condução com as questões norteadoras abertas referentes aos temas, as quais tiveram sua linguagem adaptada, sem alteração do sentido, a fim de melhorar o entendimento dos participantes devido as suas faixas etárias, sendo também realizadas as indagações necessárias.

O roteiro da entrevista possuía as questões listadas a seguir: "Alguma coisa mudou na sua vida desde que você descobriu a doença? O quê?"; "Como é o seu dia a dia? O que você faz? Vai à escola? Igreja? Encontra com amigos? Pratica algum esporte?"; "O que você quer fazer no futuro, quando for adulto? Quais são seus sonhos?".

Durante todo o curso da coleta, foi utilizado um gravador de voz a fim de registrar de maneira mais fidedigna as informações colhidas, as quais tiveram seu conteúdo transcrito com minuciosa precisão, identificando rigorosamente os elementos da linguagem dos participantes, contabilizando 1 hora e 11 minutos de áudio. Aliado aos procedimentos acima descritos, foi utilizado um diário de campo do pesquisador para registrar aspectos não verbais da comunicação, como gestos, choro, risos, entre outros.

A conclusão da coleta foi determinada pela saturação dos dados, definida pela suspensão da inclusão de novos participantes a partir do momento em que os dados passaram a apresentar certa redundância. ${ }^{(9)}$

Com o propósito de assegurar o anonimato dos participantes, durante o procedimento de coleta, eles foram identificados por pseudônimos alusivos aos personagens infantis de que mais gostavam.

Para análise dos dados, utilizou-se a análise temática descrita por Minayo. Como forma de operacionalização da técnica, realizou-se, de início, uma pré-análise, etapa na qual ocorreu a leitura exaustiva para apreensão das ideias centrais das falas, escolha dos documentos, retomada das hipóteses e objetivos iniciais, reformulando-os a partir do material da coleta, além de elaboração de indicadores que orientaram a interpretação final; constituição do corpus, agrupando as entrevistas conforme as características comuns que apresentaram; e por fim o tratamento dos resultados obtidos. ${ }^{(10)}$

Para a realização da primeira etapa da análise, os dados foram organizados e codificados com o auxílio do Atlas.ti 8.0 (Qualitative Research and Solutions), um software empregado em pesquisas qualitativas como estratégia para codificação do texto, dividindo-o em segmentos aos quais se atribuem códigos. Nessa fase, foram identificados 26 códigos, os quais geraram qua- 
tro grupos de códigos inter-relacionados, resultando cada um em uma rede que demonstra o conviver com a doença crônica.

Todo o estudo desenvolveu-se respeitando a Resolução no 466/12 do Conselho Nacional de Saúde (CNS), que trata das diretrizes e normas regulamentadoras de pesquisas envolvendo seres humanos, tendo sido aprovada pelo Comitê de Ética e Pesquisa do HUOL, por meio do Parecer no 3.301.327.

\section{Resultados}

Participaram do estudo oito crianças em idade escolar e adolescentes, dos quais sete foram meninas com idades entre 8 e 13 anos, e um menino de 8 anos; todos tinham diagnóstico médico de doença crônica. Entre esses diagnósticos, citam-se síndrome nefrótica, hepatopatia, bexiga neurogênica, fibrose cística, lúpus eritematoso sistêmico e dermatomiosite juvenil. A caracterização dos participantes do estudo consta do quadro 1.

Quadro 1. Caracterização das crianças e adolescentes participantes do estudo

\begin{tabular}{|l|c|c|c|}
\hline Pseudônimo & Idade & Sexo & Diagnóstico \\
\hline Unicórnio & 12 & Feminino & Hepatopatia \\
\hline Dora & 8 & Feminino & Síndrome nefrótica \\
\hline Barbie & 10 & Feminino & Síndrome nefrótica \\
\hline Frozen & 9 & Feminino & Bexiga neurogênica \\
\hline Ariel & 13 & Feminino & Bexiga neurogênica \\
\hline LOL & 8 & Feminino & Fibrose cística \\
\hline Homem-Aranha & 8 & Masculino & Dermatomiosite juvenil \\
\hline Snoopy & 11 & Feminino & Lúpus eritematoso sistêmico \\
\hline
\end{tabular}

Por meio da análise realizada com auxílio do software Atlas.ti 8, as seguintes categorias foram identificadas: "Modificações na vida", "Ruptura escolar", "Impactos" e "Perspectivas e sonhos". Entre as modificações na vida (Anexo 1), os participantes revelaram as restrições alimentares, como nos discursos de Unicórnio e Dora, os quais demonstram, com atitude de lamentação em suas falas, que elas não podem mais ingerir diversos alimentos agradáveis ao paladar da maioria das crianças devido aos seus problemas de saúde. Além dessas restrições, invocam também as limitações físicas, necessidades específicas ocasionadas pela doença, como o cateterismo vesical intermitente, as viagens para tratamento e as internações hospita- lares frequentes. Outro aspecto peculiar do conviver com a doença crônica foi a ruptura escolar, que envolve prejuízo da assiduidade e até mesmo perda do ano letivo (Figura 1).

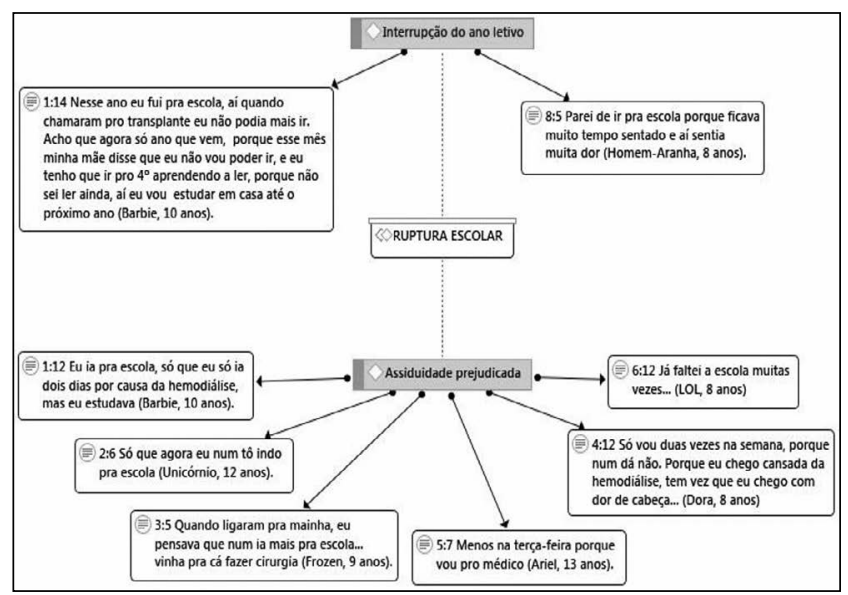

Figura 1. Ruptura escolar

A maior parte referiu não ir todos os dias à escola, por diversos motivos relacionados à doença, como consultas semanais, internações hospitalares constantes e sessões de hemodiálise. O Homem-Aranha, em seu relato com feição visivelmente triste, refere ter abandonado o ano letivo devido às dores ocasionadas pela doença, já a Barbie não pôde ir mais à escola desde que foi selecionada para a fila do transplante, tendo que permanecer no hospital durante muito tempo sem auxílio educativo, também expressando nostalgia em sua fala. Além disso, tristeza, indisposição e desânimo foram impactos causados pela descoberta da doença na vida de uma das crianças, e outra revelou sofrer bullying na escola devido ao seu corpo emagrecido, cuja perda de peso foi ocasionada pela fibrose cística, como demonstrado na figura 2.

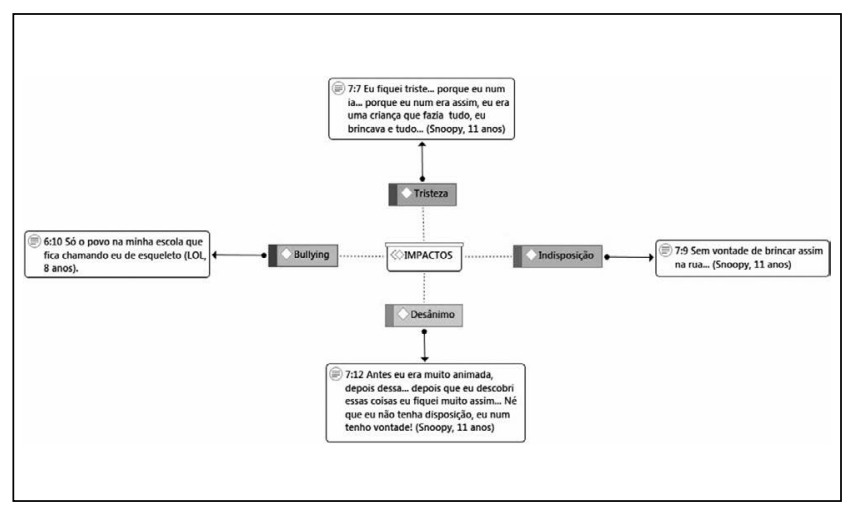

Figura 2. Impactos 
De modo pausado e ressentido, tendo lágrimas nos olhos percebidas pela mãe e pesquisadora, as falas de Snoopy demonstram caráter deprimido, assim como LOL, quando relembra sobre o preconceito e fica em silêncio por instantes. Apesar desses impasses relatados, todos os participantes revelaram ter perspectivas e sonhos para o futuro, como exibe a figura 3, ocasião da entrevista em que ostentam olhares de esperança e empolgação, pouco vistos no decorrer dela.

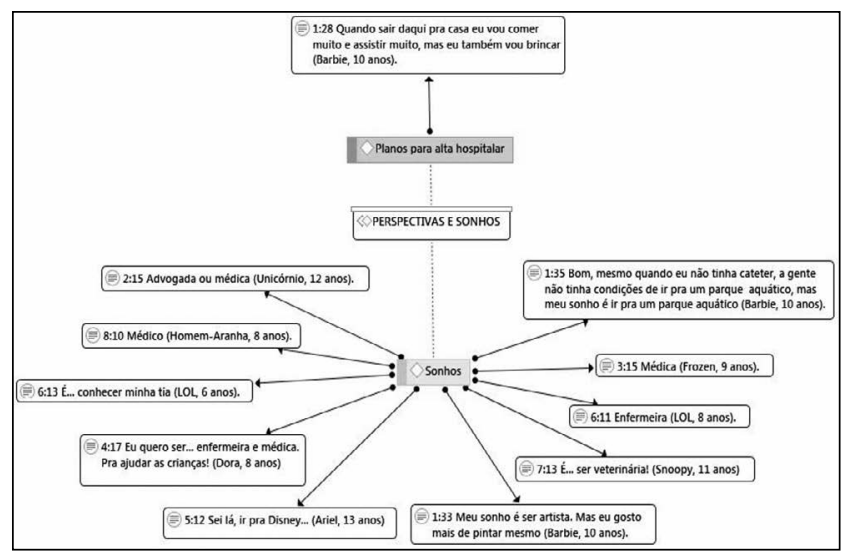

Figura 3. Perspectivas e sonhos

\section{Discussão}

Os achados deste estudo revelam que o diagnóstico de uma doença crônica pode ter impacto negativo nas vidas das crianças acometidas e de seus familiares. Estudos apontam que crianças e adolescentes podem apresentar desenvolvimento lento, acarretando alterações biológicas, psicológicas e sociais, com evidente prejuízo nas pessoas que estão em condições de maior vulnerabilidade social. ${ }^{(11)}$

No tocante a conviver com a doença crônica, os achados revelaram muitas das restrições ocasionadas pelas doenças, entre as quais se podem citar as alimentares e físicas, que interferem nas atividades cotidianas dos participantes, como brincar ou até mesmo ir à escola, o que corrobora com outros estudos..$^{(11-13)}$

Além disso, pesquisadores também apontam que diversos aspectos importantes são modificados na vida familiar, tais como o sono, relacionamentos, vida social, capacidade de trabalho, estresse, necessidades de transporte para acompanhamento da criança e sobrecarga física, emocional e financeira. ${ }^{(13,14)}$ Isso também é encontrado neste estudo, visto que as neces- sidades e sentimentos que foram relatados pelos entrevistados necessitam de um desdobramento maior por parte de suas famílias e cuidadores, exigindo um cuidado maior para não acarretar mais problemas.

Quanto à hospitalização desses indivíduos, ou mesmo ao atendimento ambulatorial, percebeu-se que esse fator apresenta vários impactos na vida deles, pois a necessidade de internação e de viagens para terem seus atendimentos causa importantes rupturas de seus cotidianos, afetando o bem-estar deles e de seus acompanhantes.

Em um estudo que buscou identificar os impactos da hospitalização em crianças com insuficiência renal crônica, foi abordado que o processo de cuidado com essa doença crônica acaba por gerar instabilidade e, consequentemente, agravos ao processo de desenvolvimento do sujeito e à sua qualidade de vida, devido às exigências espaço-temporais próprias da enfermidade crônica, que as impedem de ter uma rotina adequada. ${ }^{(15)}$

Consoante aos aspectos citados, as limitações físicas, as restrições alimentares e outras necessidades específicas que as crianças e adolescentes enfrentam devido às características de suas doenças crônicas ou pelas exigências de seus tratamentos também podem levar a sentimentos de inadequação no meio social, por eles acreditarem que são diferentes das outras pessoas na mesma faixa etária. ${ }^{(15)}$ Devido a isso, entre as rotinas de cuidados, é necessário atenção integral e contínua para o conforto da criança, com um planejamento de atividades que não as limitem.

Existem também importantes impactos sobre as crianças/adolescentes e seus pais, como os educacionais, para aqueles que, devido às suas condições, acabam por faltar na escola, nos quais se incluem aspectos neurológicos de determinadas doenças. Pode haver desafios comportamentais, como desânimo, tristeza e indisposição, da criança em relação à sua saúde ou do seu círculo social; por isso, deve-se enfatizar que é necessário procurar entender a saúde emocional das crianças e adolescentes acometidos, para que se possa fortalecer o cuidado e não prejudicar seu enfrentamento à doença. ${ }^{(16)}$

A literatura reforça que é necessária maior atenção ao acompanhamento escolar desses pacientes por parte dos profissionais de saúde e das instituições escolares, pois os prejuízos educacionais provocados pela doença, como o comprometimento da assiduidade e a interrupção do ano letivo, poderão ter repercussões negativas 
na vida adulta. ${ }^{(17,18)}$ Ter qualidade nos cuidados de saúde durante a infância pode melhorar o desempenho educacional e as perspectivas de emprego e reduzir a deficiência e a dependência na idade adulta. ${ }^{(18)}$

Por fim, no tocante às perspectivas pessoais dos participantes, houve poucos relatos relacionados aos planos para a alta hospitalar, porém houve alguns relatos sobre planos para o futuro, com citações das profissões e sonhos. Destaca-se que a maioria mencionou profissões na área da saúde, dada a sua convivência em ambientes dessa área. Além disso, surgiram desejos de viagens e ida a locais que eles nunca conheceram devido à condição social e ao uso de dispositivos médicos, retomando a temática de discursos anteriores sobre as restrições ocasionadas pelo adoecimento.

Nesse contexto, tendo em vista a fragilidade identificada nas falas dos entrevistados, o cuidado de enfermagem deve envolver os sentimentos da criança ou do adolescente e de sua família, buscando adotar estratégias de conforto para que eles enfrentem bem o conviver com o adoecimento crônico, além de procurar minimizar o estresse relacionado à hospitalização, os procedimentos relacionados à doença, entre outros aspectos que podem resultar em sofrimento psíquico.

Os profissionais de saúde podem zelar por potenciar as experiências positivas e minimizar os impactos negativos dessa condição, fazendo-se necessário escutar essas narrativas e entender o significado e os impactos em conviver com o adoecimento crônico, a fim de proporcionar uma assistência mais adequada a cada caso.

Acerca das limitações encontradas no estudo, foi identificada a falta de um espaço mais oportuno para a coleta de dados, condizente com a proposta da metodologia escolhida, para evitar a interferência de outros pares.

Sugere-se, para estudos futuros, a investigação mais aprofundada das experiências dessas famílias acerca das implicações sociais que elas vivenciam, além da abordagem das barreiras existentes para o cuidado, sobre o envolvimento da equipe multiprofissional e gestão das redes de atenção à saúde no manejo de tais problemas.

\section{Conclusão}

Por meio deste estudo, foram identificados os sentimentos relacionados a doença crônica e foi possível ter uma descrição de como é conviver com uma doença e, ao mesmo tempo, realizar as atividades da vida quotidiana. Grandes implicações foram reveladas a partir dos discursos, haja vista a fragilidade e vulnerabilidade dos participantes. Entre os inúmeros entraves que a doença ocasiona na vida dessas crianças e adolescentes, a ruptura com o meio escolar merece ênfase, pois, como é de senso comum a educação é um atributo indispensável a todo ser humano e nestes casos, visivelmente, tem sido cada vez mais dificultada. Por isso, demanda-se o estabelecimento de melhores condições de atenção educacional, como mobilizações de estratégias de articulação e comunicação entre os serviços para acompanhamento ao menor adoecido que envolva qualificação de profissionais e até mesmo melhoria na conjuntura das classes hospitalares. Diante do que foi revelado, considera-se como diferencial para a rede de atenção à saúde conhecer mais profundamente os efeitos da doença crônica na vida de sua clientela, uma vez que isso permite que a assistência seja planejada e empregada contemplando a singularidade de cada sujeito, especialmente por tratar-se dessa faixa etária, garantindo a saúde durante seu crescimento e desenvolvimento.

\section{Contribuições}

Silva JIF, Oliveira JSA, Macêdo MLAF, Dantas AKC, Fernandes SMBA e Melo FA contribuíram com a concepção do projeto, análise e interpretação dos dados, redação do artigo, revisão crítica relevante do conteúdo intelectual e aprovação da versão final a ser publicada.

\section{Referências}

1. Brasil. Ministério da Saúde. Secretaria de Atenção à Saúde. Documento de diretrizes para 0 cuidado das pessoas com doenças crônicas nas redes de atenção à saúde e nas linhas de cuidado prioritárias. Brasília (DF): Ministério da Saúde; 2013.

2. Nóbrega VM, Silva ME, Fernandes LT, Viera CS, Reichert AP, Collet N. Doença crônica na infância e adolescência: continuidade do cuidado na Rede de Atenção à Saúde. Rev Esc Enferm USP. 2017;51:e03226.

3. Hilkner SH, Beck AR, Tanaka EZ, Dini AP. Perceptions of siblings of children hospitalized due to chronic disease. Referência. 2019;4(20):77-86.

4. Parente JS, Silva FR. Perfil clínico-epidemiológico dos pacientes internados na clínica pediátrica em um hospital universitário. Rev Med UFC. 2017;57(1):10-4.

5. Silva BG, Machado AN, Nóbrega VM, Oliveira RC, Vaz EM, Collet N. Gestão do cuidado à criança/adolescente com doença crônica: (des)articulação da rede e fragmentação das ações. Rev Enferm UFSM. 2020;10(76):1-21. 
6. Pais SC, Menezes I. How do we live with chronic disease? A rights-based approach promoting the wellbeing of children with chronic disease. Ciênc Saúde Colet. 2019;24(10):3663-72.

7. Vasques RC, Mendes-Castillo AM, Bousso RS, Borghi CA, Sampaio PS. Dando voz às crianças: considerações sobre a entrevista qualitativa em pediatria. Rev Min Enferm. 2014;18(4):1016-20.

8. Meihy JC. Manual de história oral. 5a ed. São Paulo: Loyola; 2005.

9. Fontanella BJ, Luchesi BM, Saidel MG, Ricas J, Turato ER, Melo DG. Amostragem em pesquisas qualitativas: proposta de procedimentos para constatar saturação teórica. Cad Saúde Pública. 2011;27(2):389-94.

10. Minayo MC. 0 desafio do conhecimento: pesquisa qualitativa em saúde. 8a ed. São Paulo: Hucitec; 2004.

11. Gomes GC, Nornberg PK, Jung BC, Nobre CM, Rodrigues EF, Xavier DM. Doença crônica na criança: vivências da família no recebimento do diagnóstico. Rev Enferm UFPE online. 2016;10(6):4837-44.
12. Leite AC,Alvarenga WA, Machado JR, Luchetta LF, La Banca RO, Sparapani VC, et al. Crianças em seguimento ambulatorial: perspectivas do atendimento evidenciadas por entrevista com fantoche. Rev Gaúcha Enferm. 2019;40:e20180103.

13. Okido AC, Neves ET, Cavicchioli GN, Jantsch LB, Pereira FP, Lima RA. Fatores associados ao risco familiar de crianças com necessidades especiais de saúde. Rev Esc Enferm USP. 2018;52:e03377.

14. Joseph RA, Goodfellow LM, Simko LM. Parental quality of life: caring for an infant or toddler with a tracheostomy at home. Neonatal Netw. 2014;33(2):86-94.

15. Simões KC,Silva SM, Costa MP.Vozesà infância silenciada: impactos da hospitalização e hemodiálise à escolarização de crianças com doença renal crônica. Rev Educ Espec. 2020;33(e52):1-25.

16. Pickles DM, Lihn SL, Boat TF, Lannon C. A Roadmap to emotional health for children and families with chronic pediatric conditions. Pediatrics. 2020;145(2):e20191324.

17. Bell MF, Bayliss DM, Glauert R, Harrison A, Ohan JL. Chronic illness and developmental vulnerability at school entry. Pediatrics. 2016;137(5):e20152475.

18. Wijlaars LP, Gilbert R, Hardelid P. Chronic conditions in children and young people: learning from administrative data. Arch Dis Child. 2016;101(10):881-5.

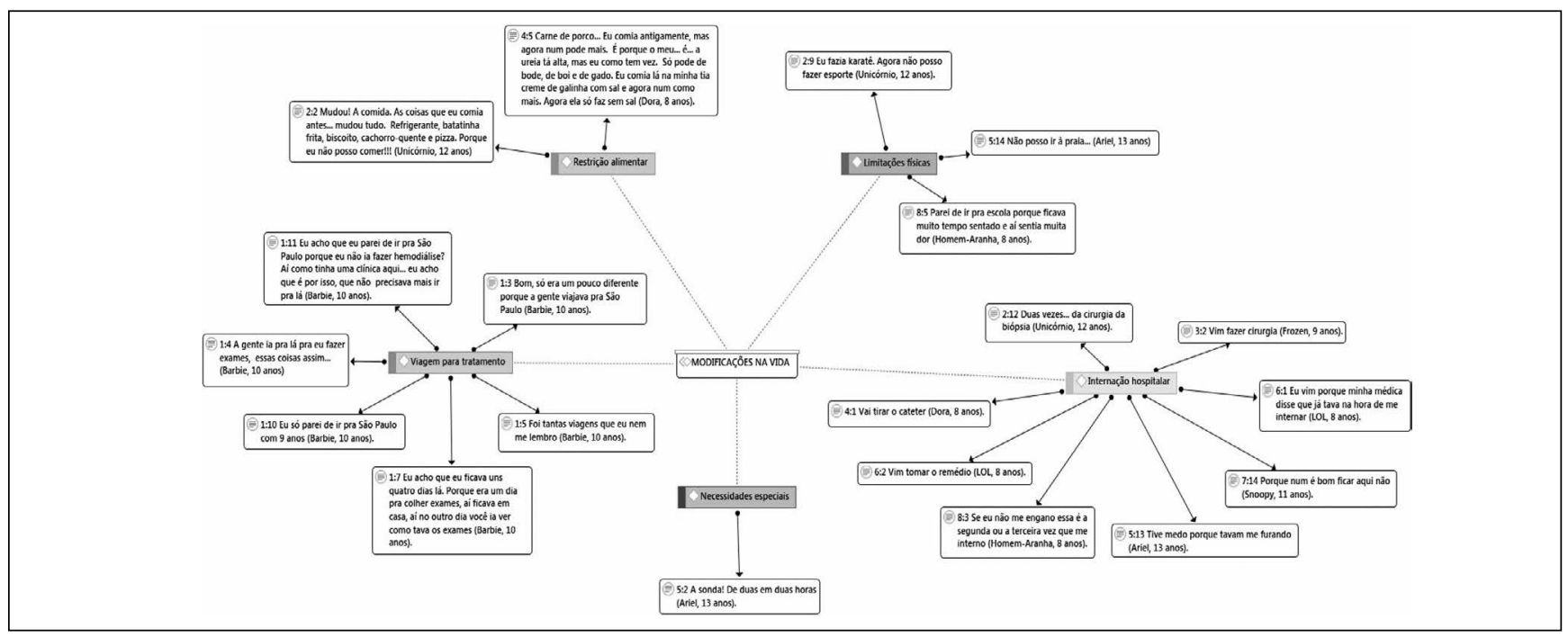

Anexo 1. Modificações na vida 